\title{
Thromboregulatory manifestations in human CD39 transgenic mice and the implications for thrombotic disease and transplantation
}

\author{
Karen M. Dwyer, ${ }^{1,2}$ Simon C. Robson, ${ }^{3}$ Harshal H. Nandurkar, ${ }^{2,4}$ Duncan J. Campbell, ${ }^{2,5}$ \\ Hilton Gock, ${ }^{1}$ Lisa J. Murray-Segal, ${ }^{1}$ Nella Fisicaro, ${ }^{1}$ Tharun B. Mysore, ${ }^{1}$ Elzbieta Kaczmarek, ${ }^{3}$ \\ Peter J. Cowan, ${ }^{1,2}$ and Anthony J.F. d'Apice ${ }^{1,2}$
}

${ }^{1}$ Immunology Research Center, St. Vincent's Hospital, Melbourne, Victoria, Australia. 'Department of Medicine, St. Vincent's Hospital, University of Melbourne, Melbourne, Victoria, Australia. ${ }^{3}$ Gastroenterology, Liver Center, Beth Israel Deaconess Medical Center, Harvard Medical School, Boston, Massachusetts, USA. ${ }^{4}$ Department of Haematology, St. Vincent's Hospital, Melbourne, Victoria, Australia. ${ }^{5}$ St. Vincent's Institute of Medical Research, Melbourne, Victoria, Australia.

\begin{abstract}
Extracellular nucleotides play an important role in thrombosis and inflammation, triggering a range of effects such as platelet activation and recruitment, endothelial cell activation, and vasoconstriction. CD39, the major vascular nucleoside triphosphate diphosphohydrolase (NTPDase), converts ATP and ADP to AMP, which is further degraded to the antithrombotic and anti-inflammatory mediator adenosine. Deletion of CD39 renders mice exquisitely sensitive to vascular injury, and CD39-null cardiac xenografts show reduced survival. Conversely, upregulation of CD39 by somatic gene transfer or administration of soluble NTPDases has major benefits in models of transplantation and inflammation. In this study we examined the consequences of transgenic expression of human CD39 (hCD39) in mice. Importantly, these mice displayed no overt spontaneous bleeding tendency under normal circumstances. The $b C D 39$ transgenic mice did, however, exhibit impaired platelet aggregation, prolonged bleeding times, and resistance to systemic thromboembolism. Donor hearts transgenic for $h C D 39$ were substantially protected from thrombosis and survived longer in a mouse cardiac transplant model of vascular rejection. These thromboregulatory manifestations in bCD39 transgenic mice suggest important therapeutic potential in clinical vascular disease and in the control of serious thrombotic events that compromise the survival of porcine xenografts in primates.
\end{abstract}

\section{Introduction}

Initially considered as a B lymphocyte marker and modulator of homotypic adhesion (1), CD39 is now recognized as a critical thromboregulatory molecule expressed on the luminal surface and caveolar microdomains of quiescent endothelial cells (ECs) $(2,3)$. When platelets come into close proximity to ECs they become unresponsive to agonists, an effect directly attributable to the nucleoside triphosphate diphosphohydrolase (NTPDase) activity of CD39 (2, $4,5)$. CD39 is the dominant vascular NTPDase and acts on ADP released from platelet-storage granules following activation. ADP is a potent agonist that triggers platelet aggregation, augments the action of other platelet agonists, and mediates adrenaline release to induce vasoconstriction (6). CD39 converts ADP to AMP, which in turn is broken down by ecto- $5^{\prime}$-nucleotidase (CD73) to adenosine $(2,6)$. The actions of adenosine on vessels and platelets oppose those of ADP. Thus, by actively suppressing platelet-EC interactions and promoting downstream generation of adenosine, CD39 acts to maintain blood fluidity and flow $(4,5)$. Although CD39 is able to act independently of prostacyclin and nitric oxide, the presence of these autacoids can enhance its biological activity.

Although damaged endothelium is a powerful stimulus of platelet activation, platelets can also interact with abnormal or activated ECs where the integrity of the endothelium is not breached (7).

Nonstandard abbreviations used: endothelial cell (EC); galactose $\alpha 1,3$-galactose ( $\alpha \mathrm{Gal})$; nucleoside triphosphate diphosphohydrolase (NTPDase).

Conflict of interest: The authors have declared that no conflict of interest exists.

Citation for this article: J. Clin. Invest. 113:1440-1446 (2004).

doi:10.1172/JCI200419560.
Activation of ECs generates a procoagulant environment through the loss of cell surface anticoagulants such as heparin sulphate and thrombomodulin $(8,9)$, promoting thrombus formation. One setting in which this process has proved to be particularly problematic is discordant xenotransplantation. The rejection of porcine xenografts by primate recipients is an extreme example of EC activation with widespread platelet sequestration and fibrin deposition (10-12). Thrombosis in vascularized xenografts is most probably exacerbated by molecular incompatibilities between porcine anticoagulant enzymes and primate coagulation factors $(13,14)$ and is frequently severe enough to lead to coagulopathy in recipients $(15,16)$. This has been observed not only with porcine solid organ transplants but also with porcine cellular transplants, where profound alterations in hemostasis and coagulation parameters are observed (17). Oxidative inactivation of CD39 (18) during ischemia/reperfusion of the transplanted organ may further contribute to the prothrombotic state. CD39 appears to be vulnerable to oxidative damage because of its extracellular location and its abundance of cysteine, methionine, and tyrosine residues $(2,19)$. CD39 activity progressively declines during the acute phases of xenograft rejection, and intravenous administration of apyrase, a soluble enzyme with action identical to CD39, inhibits platelet activation and aggregation, thereby significantly delaying the onset of rejection $(20,21)$.

Localized anticoagulation within the xenograft has obvious inherent advantages over prolonged systemic treatment, however. Adenoviral-mediated overexpression of CD39 is beneficial in the transplant setting, inhibiting vascular thrombosis and prolonging graft survival (22). We therefore generated trans- 


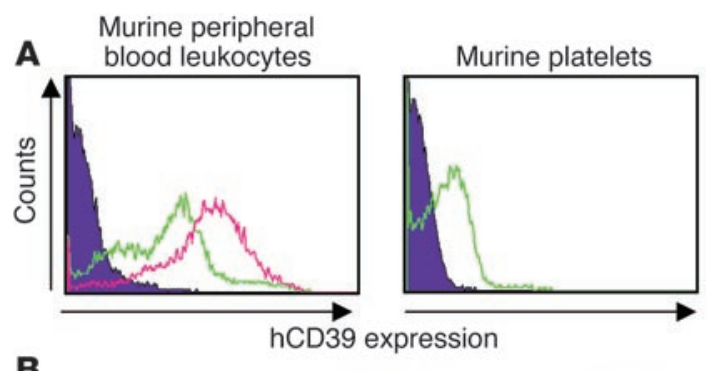

B
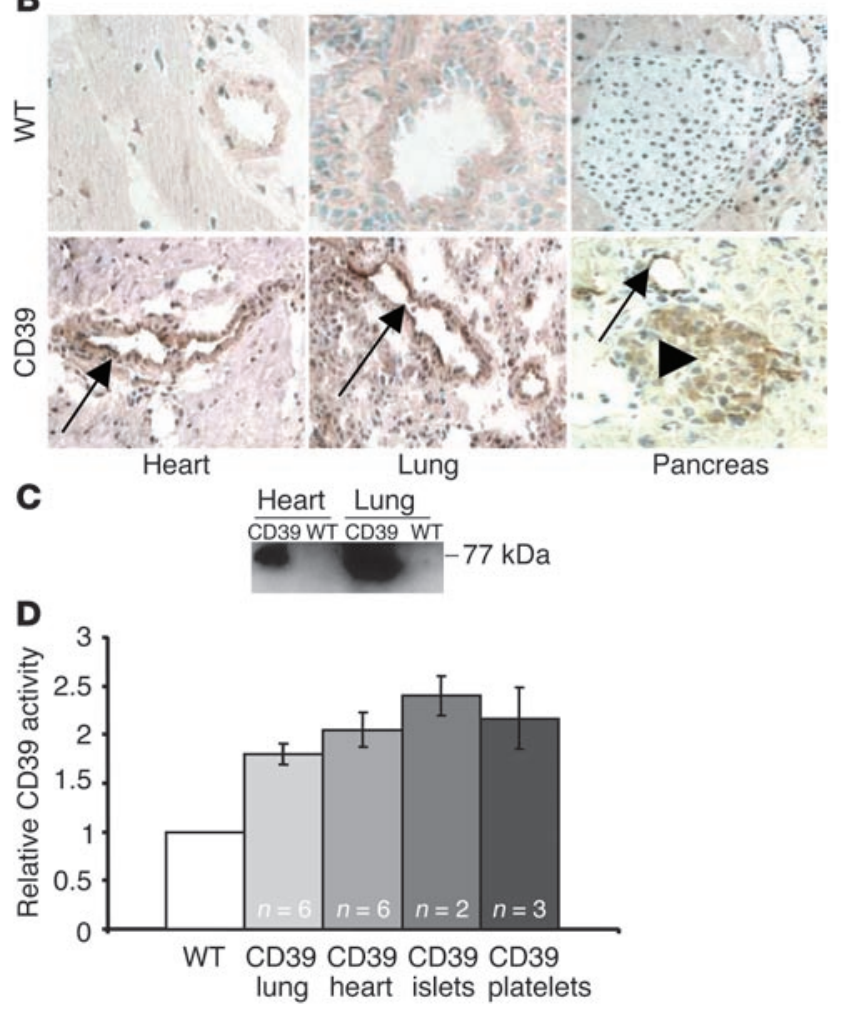

genic mice expressing human CD39 (hCD39) and demonstrate here that organs and tissues from these mice are protected from thrombosis in a transplantation setting. This is in contrast to the shortened xenograft survival times and severe end-organ damage observed in CD39-null grafts $(21,23)$.

\section{Results}

Expression of $h C D 39$ in transgenic mice. Transgenic mice were generated using a construct containing the murine $H-2 K^{b}$ (MHC class I) promoter to drive expression of hCD39. Mice were screened by flow-cytometric analysis of peripheral blood leukocytes. Two independent lines were established from founder mice strongly expressing hCD39. The transgene copy number in the two lines was estimated at 11 and 14 copies by dot blot analysis (data not shown). Both lines exhibited similar responses to thrombogenic stimuli mediated by injection of $\mathrm{ADP} /$ collagen (data not shown), indicating that the anticoagulant phenotype described in this report was unlikely to be due to transgene site-of-insertion effects. Mice expressing hCD39 were indistinguishable from nontransgenic littermates with respect to physical attributes, level of activity, and fertility. No overt bleeding tendency was associated with parturition or with routine experimental proce-

\section{Figure 1}

Human CD39 (hCD39) is widely expressed in transgenic mice. (A) Flow-cytometric analysis of leukocytes and platelets from WT (purple filled curve), heterozygote (green line), and homozygote (pink line) transgenic mice. (B) Immunohistochemical analysis (magnification $\times 40$ ) and (C) Western blot demonstrating abundant hCD39 expression in tissues of transgenic (CD39) mice but not control (WT) mice. Strong expression was detected on the vascular endothelium (arrows) and on pancreatic islets (arrowhead). (D) Increased CD39 activity in lysates of lung and heart, hand-picked islets, and washed platelets from transgenic mice were compared and expressed as the ratio to the levels in the corresponding tissues in WT mice. Data represents mean \pm SEM.

dures such as eye bleeding or tail tipping. Breeding to homozygosity resulted in a doubling of hCD39 expression (Figure 1A) without obvious detrimental effects.

Expression of hCD39 on the surface of platelets of transgenic mice (Figure 1A) did not affect absolute platelet counts $(1,000 \pm 94$ $\times 10^{9} / 1, n=3$ ). As expected, the coagulation parameters were not prolonged (international normalized ratio $0.82 \pm 0.15$, activated partial thromboplastin time $35.5 \pm 3.7$ seconds, $n=3$ ) when compared with WT mice, because these tests were conducted on platelet-free plasma. Platelet ultrastructure as determined by electron microscopy was not significantly different between transgenic and WT mice. Similar numbers of the dense granules that contain adenine nucleotides were present in both (data not shown). Consistent with our previous results using the $H-2 K^{b}$ promoter $(24,25)$, transgene expression was widespread in all tissues examined, including heart, lung, and pancreas, and was particularly strong on endothelium (Figure 1B). Correct processing of hCD39 in the tissues of transgenic mice was confirmed by Western blot analysis (Figure 1C). We then determined catalytic activity by measuring inorganic phosphate release from ADP. Total CD39 activity in heart, lung, and pancreatic islet lysates from nontransgenic mice was $10.0,10.5$, and $2.5 \mathrm{mU} / \mathrm{mg}$ protein, respectively, and in washed platelets was $120 \mathrm{mU} / 2.5 \times 10^{6}$ platelets. Corresponding samples from $b C D 39$ transgenic mice had 1.8- to 2.4-fold higher activity (Figure 1D).

hCD39 prolongs bleeding times and protects against induced thrombosis. Although transgenic mice had normal platelet numbers and coagulation parameters, in vitro studies indicated abnormal platelet aggregation responses to ADP and collagen. Whereas washed transgenic platelets reconstituted with fibrinogen and calcium demonstrated a normal and brisk response to ADP concentrations between 0.3 and $10 \mu \mathrm{M}$, attenuation of the response was observed at $0.15 \mu \mathrm{M}$ ADP (Figure 2A, left panel). The initial response of platelets from transgenic mice to collagen was also attenuated compared with that of platelets from nontransgenic mice (Figure $2 \mathrm{~A}$, right panel). Subsequently, a complete aggregatory response to both agonists was observed. To examine whether hCD39 expression affected primary hemostasis in vivo, we measured bleeding times of nicked tails suspended in saline at $37^{\circ} \mathrm{C}$. The time to hemostasis in nontransgenic mice was significantly prolonged from less than 3 minutes to a mean of 11 minutes $(P=0.03)$ by administration of apyrase (Figure $2 \mathrm{~B}$ ), indicating that this assay is appropriate for measuring functional effects of increased NTPDase activity. Transgenic mice exhibited significantly prolonged bleeding times (all more than 15 minutes, $P=0.03$ ) when compared with untreated and apyrase-treated nontransgenic mice (Figure 2B). In fact, two transgenic mice initially tested suffered hemorrhagic shock and died after 25 minutes. Subsequent experi- 
A

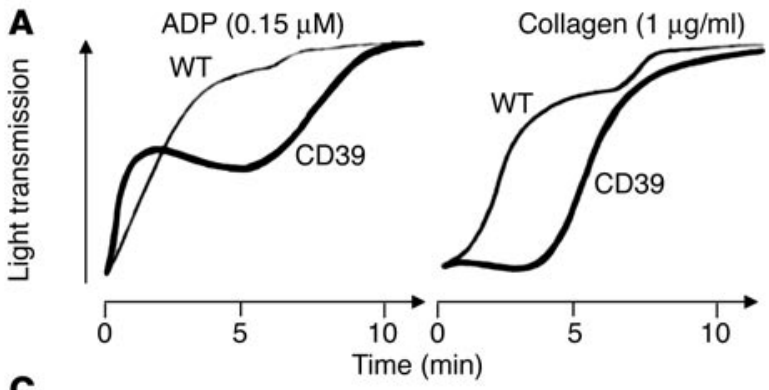

C

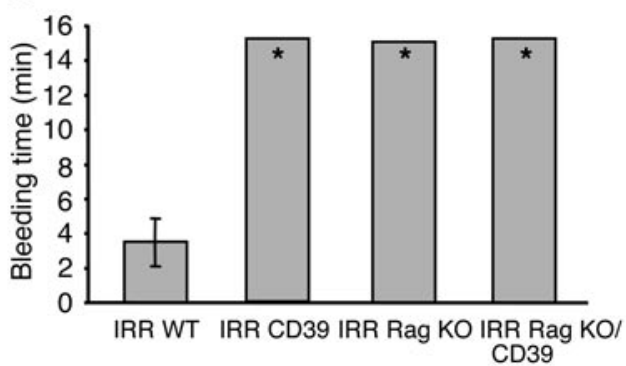

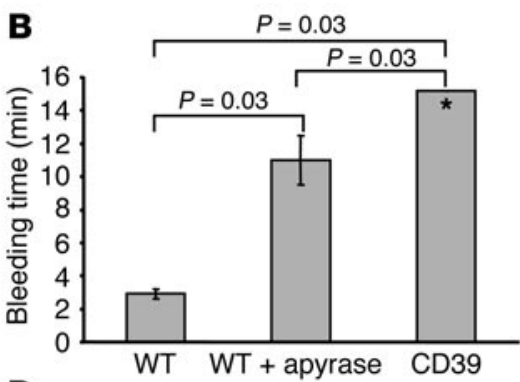

D

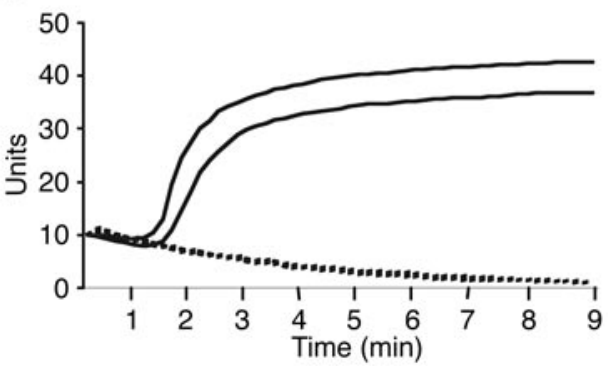

\section{Figure 2}

Expression of hCD39 in transgenic mice affects hemostasis and platelet function. (A) Platelet aggregation studies. The presence of hCD39 on the surface of platelets altered the responsiveness of platelets to agonists ADP and collagen. The initial response to both ADP and collagen was attenuated; however, a complete aggregatory response was subsequently demonstrated. Data shown are representative of three different experiments. (B) Tail-bleeding times. Expression of hCD39 significantly prolonged time to hemostasis compared with WT mice either untreated or treated with apyrase. Treatment with apyrase prolonged bleeding compared with WT control. (C) Tail-bleeding times in the adoptive transfer experiments. The presence of hCD39 either on the endothelium or blood components alone was sufficient to induce a bleeding diathesis. Experiments using transgenic mice were terminated $\left(^{*}\right)$ at 15 minutes to prevent hemorrhagic death. Data represent mean \pm SEM for six mice in each group. IRR, irradiated. (D) Whole blood aggregation. In response to collagen, WT blood (solid line) aggregated normally. This response was completely abolished in whole blood from mice transgenic for hCD39 (broken line).

ments were therefore terminated at 15 minutes by removing the tail from the saline bath and applying local pressure to the wound, which caused bleeding to cease and allowed recovery of the mice.

To determine the relative contributions of CD39 expression on endothelium and blood components to the observed bleeding phenotype, adoptive transfer experiments were performed. In the first group, $h C D 39$ transgenic mice received myeloablative irradiation before being reconstituted with nontransgenic bone marrow cells, resulting in mice with hCD39 expressed on the endothelium but not on blood components. In the second group, nontransgenic mice were reconstituted with $h C D 39$ transgenic bone marrow to generate mice with hCD39 expressed only on the blood components. Reconstitution of all blood elements was confirmed by full blood analysis with platelet counts comparable to nonirradiated mice (data not shown). Examination of bleeding times in the reconstituted mice revealed that the presence of hCD39 either on the endothelium or the blood components

\section{Figure 3}

Expression of hCD39 protects transgenic mice from death due to induced thromboembolism. (A) Survival of WT mice (white bars) and transgenic mice (gray bars) after intravenous injection with ADP, collagen, or both agents. Between five and 14 mice were examined in each treatment group and the three mice in the controls that received saline. The survival advantage afforded by hCD39 was statistically significant for collagen alone and coadministration of ADP and collagen. (B) Histological examination of the lungs from mice treated with ADP and collagen demonstrating occlusion of small vessels (arrows) in the WT but not in the transgenic lung (magnification $\times 40$ ). alone was sufficient to induce a bleeding diathesis (Figure 2C). These results were substantiated by assessment of aggregation of whole blood taken from transgenic and WT mice. The addition of
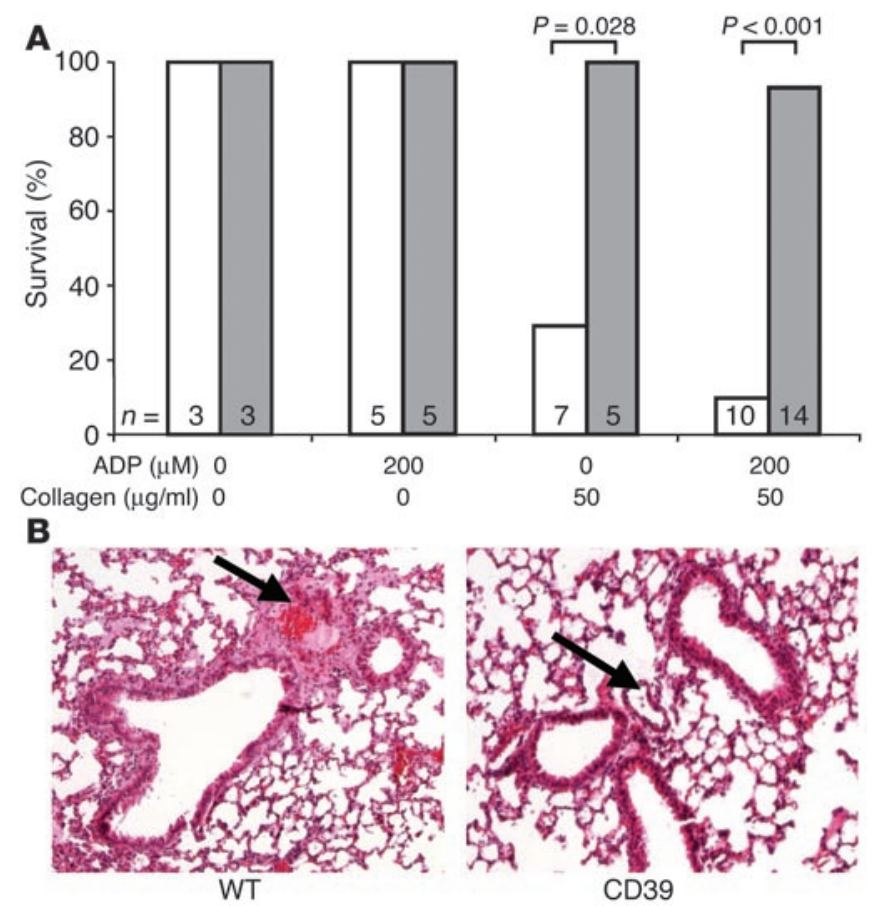
A
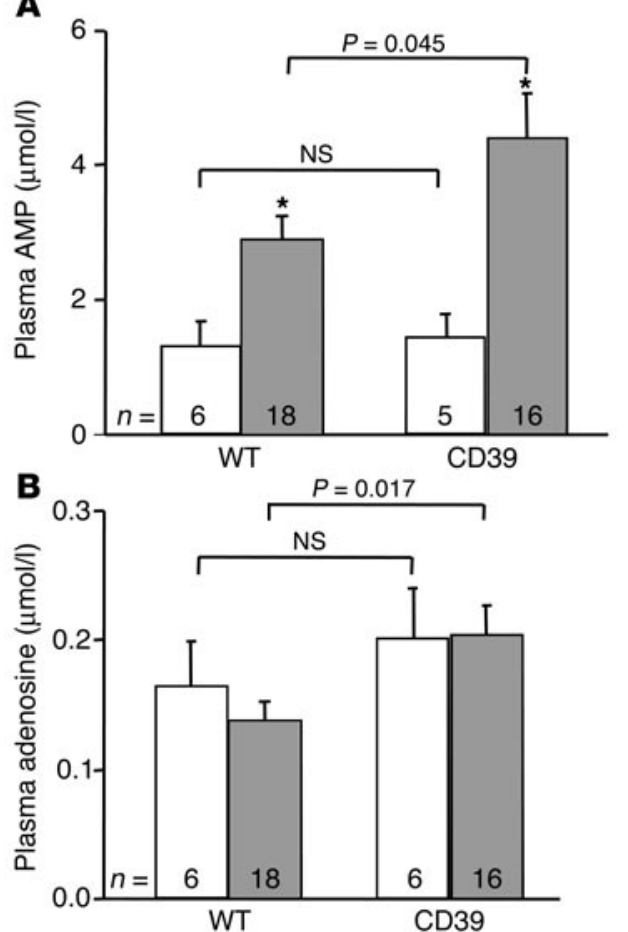

Figure 4

Expression of hCD39 elevates plasma adenosine and AMP levels. (A) The level of AMP is significantly higher ( ${ }^{*} P<0.01$ compared with basal) in both WT and $h C D 39$ transgenic mice following challenge with collagen (gray bars) as compared with basal levels (white bars), but the increase in transgenic mice is significantly greater than that observed in the WT mice. (B) Basal (white bars) adenosine levels are comparable between WT and transgenic mice, but following collagen injection (gray bars) the level of adenosine in the transgenic mice is significantly higher.

collagen $(10 \mu \mathrm{g} / \mathrm{ml})$ and $\mathrm{CaCl}_{2}(2 \mathrm{mM})$ resulted in aggregation of whole blood in WT mice, but this response was completely abrogated in the transgenic mice (Figure 2D).

To determine whether the expression of hCD39 would confer protection against systemically induced thrombosis, collagen alone or in combination with ADP was administered in the jugular vein. Collagen alone resulted in cardiorespiratory arrest and immediate death of $71 \%$ of nontransgenic mice, whereas no transgenic mice succumbed (Figure 3A). Collagen plus ADP increased mortality to $90 \%$ in nontransgenic mice, but only $7 \%$ of the transgenic mice died. Interestingly, the transgenic mice experienced a cardiorespiratory crisis immediately after injection with collagen and ADP, but the crisis was temporary in all but one mouse out of 14 tested. All animals - both WT and transgenic - survived intravenous injection of ADP alone. Histological examination showed a marked difference between lungs from treated nontransgenic and transgenic mice, the former showing thrombosis of many small vessels (Figure 3B), with an average of $22 \pm 7$ vessels occluded per field of vision in the WT lung and $4 \pm 1$ in the transgenic lung. Heavy platelet deposition was evident in and around the pulmonary veins and, in conjunction with erythrocytes, was responsible for vessel occlusion.

$A M P$ and adenosine levels are increased in $b C D 39$ transgenic mice. The coagulation and inflammatory systems are intricately associated, and this has been confirmed on a metabolic basis (26). AMP, the final product of CD39 action, is converted by ecto-5'-nucleotidase (CD73) present on the surface of endothelial cells (27) to adenosine, which has documented anti-inflammatory actions and protects the tissues from hypoxic or ischemic damage through multiple receptor subtypes $(28,29)$. Basal plasma levels of AMP and adenosine (Figure 4, A and B) were similar in $b C D 39$ transgenic mice and littermate controls. Intravenous administration of a sublethal dose of collagen significantly increased AMP but not adenosine in both groups. Importantly, the levels of AMP and adenosine after collagen treatment were significantly higher in the transgenic mice than in controls (Figure 4, A and B).

$b C D 39$ protects transgenic mouse cardiac grafts from Ab-mediated rejection. Vascular injury and thrombosis are features of vascularized xenotransplantation. We developed a mouse allograft model of xenograft rejection using recipients deficient in expression of galactose $\alpha 1,3$-galactose ( $\alpha \mathrm{Gal})$, the major porcine epitope recognized by the human immune system. Eighty-seven percent of heterotopic cardiac transplants from $\alpha \mathrm{Gal}$-positive $b C D 39$-negative donors to $\alpha \mathrm{Gal}^{-/-}$recipients were rejected within 24 hours of intravenous injection of anti- $\alpha \mathrm{Gal} \operatorname{IgG} 1 \mathrm{mAb}$ (Table 1). Rejection was characterized by widespread intravascular thrombosis and a striking infiltrate of platelets, particularly concentrated within smaller blood vessels (Figure 5). This was associated with destruction of the cardiac ultrastructure with compete separation of cardiac myofibrils due to interstitial hemorrhage. In contrast, only $15 \%$ of $\alpha$ Gal-positive $h C D 39$-expressing cardiac allografts failed within 24 hours of anti- $\alpha$ Gal injection, the remaining grafts continuing to function and showing markedly less platelet deposition throughout the myocardium and within smaller blood vessels (Table 1 and Figure 5) and preservation of cardiac architecture. These grafts continued to beat for up to 6 days after injection (9 days after transplantation), at which point they succumbed to allograft rejection. Histologically, $h C D 39$ expression was undiminished on the endothelium of cardiac blood vessels in grafts failing up to 6 days after injection (data not shown).

\section{Discussion}

CD39 is an important regulator of platelet function, and we demonstrate here that expression of hCD39 in transgenic mice results in a modified hemostatic system with impaired platelet aggregation and prolonged bleeding times. This antithrombotic phenotype was also manifested in $b C D 39$ transgenic mice by resistance to thrombogenic stimuli fatal to nontransgenic mice. The transgenic mice displayed no overt bleeding tendency under normal circumstances, but transection of the tail under anesthesia resulted in excessive bleeding, which, if unchecked, led to death. This effect of anesthesia on the bleeding tendency may be due to inhibition of the sympathetic nervous system. Activation of the sympathetic nervous system causes release of noradrenaline and consequently platelet activation and vasoconstriction. In anesthe-

\section{Table 1}

Survival of $\alpha \mathrm{Gal}-$ positive to $\alpha \mathrm{Gal}{ }^{-/}$cardiac allografts (days) following injection of $0.075 \mathrm{mg}$ anti- $\alpha \mathrm{Gal}$ lgG1 mAb

$\begin{array}{lr}\text { WT donor } & <1<1,<1,<1,<1,<1,<1,3 \\ \text { CD39 transgenic donor } & <1,<1,2,3,3,5,6,6,6\end{array}$

$P=0.011$. Numbers in the right-hand column represent the day after $\mathrm{mAb}$ treatment at which individual cardiac grafts ceased to beat. 

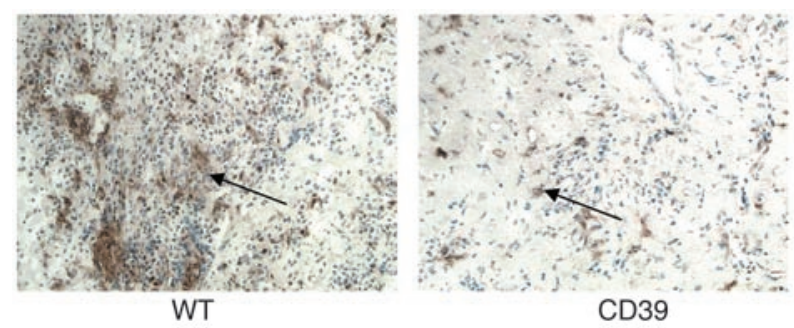

Figure 5

Expression of hCD39 protects hearts from rejection in an allograft model of cardiac xenotransplantation. Immunohistochemical analysis of grafts removed at 24 hours after Ab injection, demonstrating preserved morphology and minimal platelet deposition in capillaries (arrow) in transgenic grafts. In contrast, WT grafts show a heavy platelet infiltrate (arrow) and destruction of myocardial architecture (magnification $\times 20$ ).

tized $h C D 39$ transgenic mice, an increased local ratio of adenosine to ADP resulting from elevated CD39 expression may allow adenosine to act unopposed on the vascular smooth muscle of blood vessels, causing vasodilation. In addition, adenosine inhibits platelet reactivity by elevating platelet cAMP and may therefore exert a direct antiaggregatory effect on the platelet plug, further limiting the ability of the anesthetized transgenic mice to obtain primary hemostasis. Reconstitution experiments demonstrated that increased CD39 expression on either the endothelium or the blood components was sufficient to induce the bleeding diathesis. Recent work by Sesti et al. (7) has demonstrated the ability of ATP to augment or inhibit noradrenaline release, depending on the local concentration of ATP. ATP and noradrenaline are stored together in adrenergic nerve cell vesicles and are coreleased during sympathetic neurotransmission (30). In the cardiac sympathetic nervous system, ATP facilitates noradrenaline release through activation of $\mathrm{P} 2 \mathrm{X}$ receptors and inhibits noradrenaline release through interaction with $\mathrm{P} 2 \mathrm{Y}$ receptors (7). As with administration of soluble CD39 (7), $b$ CD39 transgenic mice with enhanced CD39 activity may generate lower ATP concentrations and reduced noradrenaline release, thereby exaggerating the bleeding diathesis.

Paradoxically, CD39-null mice have a bleeding phenotype similar to that of $h C D 39$ transgenic mice, but this is secondary to purinoreceptor desensitization and is correctable with prior administration of exogenous apyrase (23). Interestingly, a different CD39-null mouse line has a normal bleeding phenotype but cannot withstand platelet deposition induced by experimental cerebral infarction, the effects of which can be reversed with the administration of soluble CD39 (31). The purinergic $\mathrm{P}_{1} \mathrm{Y}_{1}$ receptor is integral to ADP function, and $\mathrm{P}_{2} \mathrm{Y}_{1}$-deficient mice also exhibit prolonged bleeding times due to the lack of a target for $\operatorname{ADP}(32,33)$.

Platelets normally aggregate upon exposure to agents such as ADP and collagen, provoking a primary response that is augmented by a secondary response triggered by the release of intracellular ADP. ADP release from platelet granules is integral to amplifying and propagating platelet activation, aggregation, and recruitment in response to the initiating stimulus $(2,34)$, with the resultant platelet plug exposing a phospholipoprotein surface that provides a scaffolding upon which coagulation proteins assemble for the promotion of thrombin formation and conversion of fibrinogen to fibrin. Collagen interacts directly with two platelet membrane glycoproteins to induce activation and aggregation via an ADP-dependent mechanism $(35,36)$.
Transfected COS-7 cells expressing high levels of hCD39 completely inhibited platelet aggregation in response to ADP or collagen (2). Washed platelets from $h C D 39$ transgenic mice expressing modest ADPase activity exhibited a less-pronounced dampening of the aggregatory response, with a lack of primary response to low-dose ADP, and the normal delayed primary response to collagen was prolonged. It should be noted that the handling associated with preparation of the platelets might have heightened the degree of sensitivity to the triggering stimulus. Nevertheless, the results suggest that expression of hCD39 on the platelet surface was sufficient to hydrolyze ADP at physiological concentrations, but was overwhelmed by the release of intracellular ADP, resulting in delayed but ultimately complete aggregation in vitro. Platelets from $\mathrm{P}_{2} \mathrm{Y}_{1}$ receptor-deficient mice fail to aggregate in response to low-dose ADP or collagen (32), and platelets from CD39-null mice are either hyporeactive (23) or responsive to ADP and collagen (31), depending on the line. Interestingly, whole blood from $h C D 39$ transgenic mice demonstrated complete unresponsiveness to high-dose collagen, presumably because of the contribution of strong hCD39 expression on leukocytes.

The ability of $h C D 39$ transgenic mice to survive systemic injection of $\mathrm{ADP} /$ collagen suggests that they are at least partially protected against ADP-induced platelet activation in vivo. Injection of ADP alone had no demonstrable effect on transgenic or nontransgenic mice, presumably because of efficient inactivation by endogenous CD39. Transgenic mice suffered a transient cardiorespiratory arrest following injection of collagen alone or in combination with ADP, but their subsequent recovery indicated protection against exogenous and endogenous ADP. The cardiorespiratory crisis may have been due to the release of nitric oxide and/or prostacyclin. $\mathrm{P}_{2} \mathrm{Y}_{1}$-deficient mice display a similar protection against collagen- and ADP-induced thromboembolism $(32,33)$. The survival advantage of the $b C D 39$ transgenic mice was most likely due to the ability to metabolize the ADP released by the collagen and the ADP itself, combined with the cytoprotective effects of adenosine generated by the action of endothelial 5'-nucleotidase. CD39-null mice are very sensitive to ischemia-reperfusion injury because of their inability to generate adenosine in the local vascular bed, and they are protected by apyrase treatment (23).

AMP, the final product of CD39 action, was elevated following challenge with collagen in both transgenic and WT mice, but the induced level was significantly higher in the transgenic mice. Although it might be predicted that the conversion of AMP to adenosine by endothelial 5 '-nucleotidase would result in elevated adenosine in response to collagen, this was not observed in transgenic or WT mice. This may reflect the rapid clearance of adenosine from the circulation by erythrocytes (37) and the fact that blood was drawn for analysis at a site distal to collagen administration.

The introduction of cyclosporine has significantly improved the 1-year allograft survival through the reduction of acute rejection episodes (38). Chronic rejection, however, a process characterized by smooth muscle proliferation and consequent organ ischemia, remains the most important cause of long-term allograft loss. In contrast, the barrier currently facing xenotransplantation of solid vascularized organs is acute vascular rejection. Rejected vascularized xenografts exhibit widespread vascular injury with extensive fibrin and platelet deposition. Unregulated platelet activation leads to the recruitment of several systems that may be involved in the pathogenesis of this 
injury $(10,11,39)$. Inflammation is a prominent feature of both allograft and xenograft rejection, and targeting this system is an important function of current immunosuppressive regimens. Cardiac xenografts from CD39-null mice in rats failed more rapidly and showed increased fibrin deposition compared with WT control grafts, indicating a role for CD39 in protection against rejection (23). We confirmed this role using a model of Ab-mediated xenograft rejection that is characterized by rejection within 24 hours associated with significant thrombosis within the graft. Expression of hCD39 on donor hearts attenuated platelet deposition and small vessel thrombosis, thereby improving perfusion. The relative contributions of the anticoagulant and anti-inflammatory properties of hCD39 in preventing rapid rejection are yet to be determined. Previous work (18) has documented loss of CD39 activity with xenograft rejection. We demonstrated persistent CD39 expression up to 9 days after transplantation ( 6 days after injection of $\mathrm{Ab}$ ), consistent with studies on CD39 adenoviral-infected cardiac grafts that demonstrated that a decline in CD39 activity immediately after transplantation was reversed 3 days later (22). Recovery of CD39 activity was not observed in control grafts (22).

Our work suggests that hCD39, in addition to preventing induced thrombosis, confers a survival advantage to xenografts and affirms the importance of the coagulation system and particularly platelet activation in the pathogenesis of xenograft rejection. Our observations open up therapeutic possibilities for the use of porcine organs and cells transgenic for hCD39 in xenotransplantation, the success of which to date has been hampered by thrombotic complications.

\section{Methods}

Transgenic mice. The cDNA for $b C D 39$ was cloned as a $1.7-\mathrm{kb}$ EcoRI fragment from pcDNA3/CD39 (2) into a plasmid containing the murine $H-2 K^{b}$ promoter (16). A $7.2-\mathrm{kb}$ fragment comprising the promoter, intron, $\mathrm{cDNA}$, and polyadenylation signal was excised using BssHII and microinjected into fertilized CBA/ C57BL6 mouse oocytes. Genomic DNA was prepared from tail tips of progeny mice using the QIAamp DNA Mini Kit (QIAGEN, Melbourne, Australia), digested with EcoRI, and screened for incorporation of the transgene by Southern blot analysis using the $1.7-\mathrm{kb}$ EcoRI CD39 cDNA above as a probe. Mice were bred and housed in an approved animal facility at the Immunology Research Center, Melbourne, Australia, and all experiments were carried out with the approval of the Animal Ethics Committee of St. Vincent's Hospital, Melbourne. Controls used in experiments were nontransgenic littermates.

Flow cytometry. Mouse peripheral blood leukocytes or platelets were incubated with fluorescein-labeled mouse anti-human CD39 mAb (Ancell Corp., Bayport, Minnesota, USA) and analyzed on a FACScalibur flow cytometer (Becton-Dickinson and Co., Franklin Lakes, New Jersey, USA).

Immunohistochemistry. Fresh-frozen tissue sections were incubated with the anti-human CD39 Ab followed by peroxidaseconjugated sheep anti-fluorescein polyclonal Ab. Staining was developed using 3,3'-diaminobenzidine tetrahydrochloride and counterstained with hematoxylin.

Western blotting. Tissue lysate proteins $(50 \mu \mathrm{g})$ were electrophoresed under nonreducing conditions and transferred to a PVD membrane, which was incubated with anti-human $\mathrm{CD} 39 \mathrm{Ab}$ at $4{ }^{\circ} \mathrm{C}$ overnight. The membrane was washed and incubated with HRP- conjugated rabbit anti-mouse IgG, and the signal was developed by ECL (Amersham Biosciences, Amersham, United Kingdom).

Hematological parameters. Platelet counts and coagulation parameters were measured using EDTA- and citrate-treated blood, respectively, on a full blood count analyzer (Sysmex SE9000; TOA Medical Electronics Co., Kobe, Japan). Platelet aggregation was studied using $200 \times 10^{3}$ washed platelets $/ \mu \mathrm{l}$ in $500 \mu \mathrm{l}$ of Tyrode's buffer at $37^{\circ} \mathrm{C}$. Aggregation was measured by light scattering on a platelet aggregation chromogenic kinetics system (PACKS-4; Helena Laboratories, Beaumont, Texas, USA). Whole blood aggregation was studied using 1:10 vol/vol citrated venous mouse blood diluted 1:2 in normal saline, and impedance was measured on a whole blood lumiaggregometer (Chrono-Log Corp., Havertown, Pennsylvania, USA).

Electron microscopy. Washed platelets were analyzed by electron microscopy at the University of Melbourne, Australia, Department of Botany.

CD39 activity assay. Tissue lysates or platelets were diluted in $100 \mu \mathrm{l}$ of assay buffer $(8 \mathrm{mM} \mathrm{CaCl} 2 / 5 \mathrm{mM}$ tetramisole $/ 50 \mathrm{mM}$ Tris-Base $/ 50 \mathrm{mM}$ imidazole/150 mM NaCl, $\mathrm{pH} 7.5)$. Twenty-five microliters of $3 \mathrm{mM}$ ADP was added, and after incubation at $36^{\circ} \mathrm{C}$ for 20 minutes the reaction was terminated by the addition of $25 \mu \mathrm{l}$ of reagent $(2.5 \mathrm{ml} 7.5 \%$ molybate $/ 10 \mathrm{ml}$ malachite green hydrochloride in sulfuric acid/0.2 $\mathrm{ml} 11 \%$ Tween). After 20 minutes, the absorbance at $620 \mathrm{~nm}$ was measured using a FLUOstar Galaxy plate reader (BMG Labtechnologies, Offenburg, Germany). One unit of ATPDase activity corresponds to the release of $1 \mu \mathrm{mol} \mathrm{P}_{\mathrm{i}} / \mathrm{min}$ at $37^{\circ} \mathrm{C}(40)$.

Bleeding times. Mice were anesthetized with ketamine and xylazine $(0.1 \mathrm{ml} / \mathrm{g}$ body weight), and the tail was transected $5 \mathrm{~mm}$ from the tip. The tail was then suspended in saline warmed to $37^{\circ} \mathrm{C}$, and the time to cessation of bleeding was recorded.

Adoptive transfer studies. Mice transgenic for $h C D 39$ were bred onto the immunodeficient Rag1 KO background. Transgenic mice received myeloablative irradiation (two courses of 5.5 Gy, 3 hours apart) before being reconstituted with $0.8 \times 10^{6}$ nontransgenic bone marrow cells administered by tail vein injection. Nontransgenic recipient mice received the same treatment but were reconstituted with $h C D 39$ transgenic bone marrow cells. The appropriate controls (transgenic to transgenic and WT to WT) were performed in parallel. Following cervical dislocation, bone marrow was harvested from donor mice by flushing the long bones with mouse tonicity PBS. The marrow was filtered and centrifuged, and the cells were counted and injected in a $200 \mu \mathrm{l} \mathrm{vol-}$ ume. Reconstitution was confirmed at 3 weeks after transplant with a full blood count and flow-cytometric analysis on peripheral blood leukocytes for hCD39.

Induced thromboembolism model. Four microliters per gram of body weight of a saline-based solution containing $200 \mu \mathrm{M}$ ADP and/or $50 \mu \mathrm{g} / \mathrm{ml}$ collagen was administered to anesthetized mice through the jugular vein. Survival at 1 hour was recorded (32). Lungs were examined histologically with H\&E, specific platelet (anti-CD41), and fibrin (Martius scarlet blue) staining. Quantitation of occluded vessels was determined by counting the number of occluded vessels per field of vision $(\times 20$ magnification). Eight fields were examined for both the WT and transgenic mice.

Adenosine and AMP measurements. The methodology was based on those described by Saadjian et al. (41). Blood $(0.5 \mathrm{ml})$ was drawn from the inferior vena cava of anesthetized mice into a syringe containing $1 \mathrm{ml}$ stop solution containing $0.2 \mathrm{mmol} / \mathrm{l}$ dipyridamole, 
$5 \mathrm{mmol} / 1$ erythro-9-(2-hydroxy-3-nonyl)-adenine, and $4.2 \mathrm{mmol} / 1$ EDTA in $0.15 \mathrm{~mol} / 1$ sodium chloride. Following centrifugation, 1 $\mathrm{ml}$ of the supernatant was then added to $0.05 \mathrm{ml} 11.7 \mathrm{~mol} / \mathrm{l}$ perchloric acid. The precipitated plasma proteins were then centrifuged to create sediments, and perchloric acid was extracted from the supernatant with $2 \mathrm{ml}$ chloroform/tri-n-octylamine (78:22, vol/vol). The supernatant was lyophilized, dissolved in $10 \mathrm{mM}$ potassium phosphate buffer, $\mathrm{pH} 7.0$, and then run on HPLC. Adenosine and AMP were identified from their elution positions as determined with standards obtained from Sigma-Aldrich (St. Louis, Missouri, USA).

Cardiac allograft, psendodiscordant xenograft model. The $\alpha \mathrm{Gal}$-positive CBA/C57BL6 donor hearts (heterozygous for $b C D 39$ transgenic or nontransgenic littermates) were harvested and grafted into $\mathrm{Gal}^{-/-} \mathrm{BALB} / \mathrm{c}$ recipients. At 3 days after surgery, the recipient was injected with $75 \mu \mathrm{g}$ anti- $\alpha \mathrm{Gal}$ IgG1 mAb through the tail vein. The transplanted heart was monitored by daily palpation. The heart was deemed rejected when the cardiac beat was absent.

Statistics. ANOVA was used for comparison of plasma AMP and adenosine levels. All other statistical calculations used the Mann-Whitney test.

1. Maliszewski, C.R., et al. 1994. The CD39 lymphoid cell activation antigen. Molecular cloning and structural characterization. J. Immunol. 153:3574-3583.

2. Kaczmarek, E., et al. 1996. Identification and characterization of CD39/vascular ATP diphosphohydrolase. J. Biol. Chem. 271:33116-33122.

3. Goepfert, C., et al. 2000. CD39 modulates endothelial cell activation and apoptosis. Mol. Med. 6:591-603.

4. Marcus, A.J., et al. 1991. Inhibition of platelet function by an aspirin-insensitive endothelial cell ADPase. Thromboregulation by endothelial cells. J. Clin. Invest. 88:1690-1696.

5. Marcus, A.J., et al. 1995. Thrombosis and inflammation as multicellular processes: significance of cell-cell interactions. Thromb. Haemost. 74:213-217.

6. Zimmermann, H. 1992. 5'-Nucleotidase: molecular structure and functional aspects. Biochem. $J$. 285:345-365.

7. Sesti, C., et al. 2002. Ectonucleotidase in cardiac sympathetic nerve endings modulates ATP-mediated feedback of norepinephrine release. J. Pharmacol. Exp. Ther. 300:605-611.

8. Platt, J.L., et al. 1990. Release of heparan sulfate from endothelial cells. Implications for pathogenesis of hyperacute rejection. J. Exp. Med. 171:1363-1368.

9. Moore, K.L., Esmon, C.T., and Esmon, N.L. 1989. Tumor necrosis factor leads to the internalization and degradation of thrombomodulin from the surface of bovine aortic endothelial cells in culture. Blood. 73:159-165.

10. Bach, F.H., et al. 1994. Endothelial cell activation and thromboregulation during xenograft rejection. Immunol. Rev. 141:5-30.

11. Bach, F.H., Winkler, H., Ferran, C., Hancock, W.W., and Robson, S.C. 1996. Delayed xenograft rejection. Immunol. Today. 17:379-384.

12. Robson, S.C., et al. 1995. Role of endothelial cells in transplantation. Int. Arch. Allergy Immunol. 106:305-322.

13. Siegel, J.B., et al. 1997. Xenogeneic endothelial cells activate human prothrombin. Transplantation. 64:888-896.

14. Kopp, C.W., et al. 1997. Effect of porcine endothelial tissue factor pathway inhibitor on human coagulation factors. Transplantation. 63:749-758.

15. Ierino, F.L., et al. 1998. Disseminated intravascular coagulation in association with the delayed rejection

\section{Acknowledgments}

We thank Helen Barlow and staff at the Immunology Research Center animal house for the generation and maintenance of transgenic mice; Robert Andrews, Monash University, Australia, for his assistance with whole blood aggregometry; and the Walter and Eliza Hall Institute (WEHI), Melbourne, Australia, for the use of irradiation facilities. This project was funded by the National Health and Medical Research Council (NHMRC) of Australia and NIH grants HL57307 and HL-63972. K.M. Dwyer is a recipient of a Medical Postgraduate Research Scholarship from the NHMRC, Australia, and D.J. Campbell is a recipient of a Career Development Fellowship (Award CR 02M 0829) from the National Heart Foundation of Australia.

Received for publication July 22, 2003, and accepted in revised form March 16, 2004.

Address correspondence to: Anthony J.F. d'Apice, Immunology Research Centre, St. Vincent's Hospital, Melbourne, Post Office Box 2900, Fitzroy, Victoria 3065, Australia. Phone: 613-9288-3140; Fax: 613-9288-3151; E-mail: dapice@svhm.org.au. of pig-to-baboon renal xenografts. Transplantation. 66:1439-1450.

16. Cowan, P.J., et al. 2000. Renal xenografts from triple-transgenic pigs are not hyperacutely rejected but cause coagulopathy in non-immunosuppressed baboons. Transplantation. 69:2504-2515.

17. Buhler, L., et al. 2000. Coagulation and thrombotic disorders associated with pig organ and hematopoietic cell transplantation in nonhuman primates. Transplantation. 70:1323-1331.

18. Robson, S.C., et al. 1997. Loss of ATP diphosphohydrolase activity with endothelial cell activation. J. Exp. Med. 185:153-163.

19. Wang, T.F., and Guidotti, G. 1996. CD39 is an ecto(Ca2+,Mg2+)-apyrase. J. Biol. Chem. 271:9898-9901.

20. Koyamada, N., et al. 1996. Apyrase administration prolongs discordant xenograft survival. Transplantation. 62:1739-1743.

21. Imai, M., et al. 1999. Modulation of nucleoside [correction of nucleotide] triphosphate diphosphohydrolase-1 (NTPDase-1)cd39 in xenograft rejection. Mol. Med. 5:743-752.

22. Imai, M., et al. 2000. Recombinant adenoviral mediated CD39 gene transfer prolongs cardiac xenograft survival. Transplantation. 70:864-870.

23. Enjyoji, K., et al. 1999. Targeted disruption of cd39/ATP diphosphohydrolase results in disordered hemostasis and thromboregulation. Nat. Med. 5:1010-1017.

24. Chen, C.-G., et al. 1996. Reduction in Gal- $\alpha 1,3-\mathrm{Gal}$ epitope expression in transgenic mice expressing human H-transferase. Xenotransplantation. 3:69-75.

25. Cowan, P.J., et al. 2002. Protective effects of recombinant human antithrombin III in pig-to-primate renal xenotransplantation. Am. J. Transplant. 2:520-525.

26. Marcus, A.J., et al. 1982. Formation of leukotrienes and other hydroxy acids during platelet-neutrophil interactions in vitro. Biochem. Biophys. Res. Commun. 109:130-137.

27. Zimmermann, H., and Braun, N. 1999. Ecto-nucleotidases-molecular structures, catalytic properties, and functional roles in the nervous system. Prog. Brain Res. 120:371-385.

28. Liang, B.T., and Jacobson, K.A. 1998. A physiological role of the adenosine A3 receptor: sustained cardioprotection. Proc. Natl. Acad. Sci. U. S. A. 95:6995-6999.

29. Sato, T., Sasaki, N., O'Rourke, B., and Marban, E. 2000. Adenosine primes the opening of mitochondrial ATP-sensitive potassium channels: a key step in ischemic preconditioning? Circulation. 102:800-805

30. von Kugelgen, I., Allgaier, C., Schobert, A., and Starke, K. 1994. Co-release of noradrenaline and ATP from cultured sympathetic neurons. Neuroscience. 61:199-202.

31. Pinsky, D.J., et al. 2002. Elucidation of the thromboregulatory role of CD39/ectoapyrase in the ischemic brain. J. Clin. Invest. 109:1031-1040. doi:10.1172/JCI200210649.

32. Fabre, J.E., et al. 1999. Decreased platelet aggregation, increased bleeding time and resistance to thromboembolism in P2Y1-deficient mice. Nat. Med. 5:1199-1202.

33. Leon, C., et al. 1999. Defective platelet aggregation and increased resistance to thrombosis in purinergic P2Y(1) receptor-null mice. J. Clin. Invest. 104:1731-1737.

34. Kroll, M.H., and Schafer, A.I. 1989. Biochemical mechanisms of platelet activation. Blood. 74:1181-1195.

35. Van Deijnen, J.H., van Suylichem, P.T., Wolters, G.H., and van Schilfgaarde, R. 1994. Distribution of collagens type I, type III and type V in the pancreas of rat, dog, pig and man. Cell Tissue Res. 277:115-121.

36. van Suylichem, P.T., van Deijnen, J.E., Wolters, G.H., and van Schilfgaarde, R. 1995. Amount and distribution of collagen in pancreatic tissue of different species in the perspective of islet isolation procedures. Cell Transplant. 4:609-614.

37. Gewirtz, H., Brown, P., and Most, A.S. 1987. Measurement of plasma adenosine concentration: methodological and physiological considerations. Proc. Soc. Exp. Biol. Med. 185:93-100.

38. Merion, R.M., White, D.J., Thiru, S., Evans, D.B., and Calne, R.Y. 1984. Cyclosporine: five years' experience in cadaveric renal transplantation. $N$. Engl.J. Med. 310:148-154.

39. Candinas, D., et al. 1996. Inhibition of platelet integrin GPIIbIIIa prolongs survival of discordant cardiac xenografts. Transplantation. 62:1-5.

40. Imai, M., et al. 1999. Suppression of ATP diphosphohydrolase/CD39 in human vascular endothelial cells. Biochemistry. 38:13473-13479.

41. Saadjian, A.Y., et al. 2002. Role of endogenous adenosine as a modulator of syncope induced during tilt testing. Circulation. 106:569-574. 